\title{
Distributed digital libraries platform in the PIONIER network
}

\author{
Cezary Mazurek, Tomasz Parkoła, Marcin Werla \\ Poznan Supercomputing and Networking Center \\ Noskowskiego 10, 61-704 Poznań, Poland \\ \{mazurek,tparkola,mwerla\}@man.poznan.pl
}

\section{Demo description}

The dLibra Digital Library Framework (http://dlibra.psnc.pl/) is a Polish digital library software platform developed by Poznan Supercomputing and Networking Center as a part of the PIONIER programme (http://www.pionier.gov.pl/). The dLibra project was started in 1999, as a part of research in the field of digital libraries started in PSNC in 1996. The developed platform is currently the most popular digital library framework in Poland. It has been deployed for the first time in the Digital Library of the Wielkopolska Region (http://www.wbc.poznan.pl/, since 2002) . Currently it is used in more than 8 digital libraries in Poland and 5 of them are regional digital libraries.

All these libraries create together a platform of distributed digital libraries in the PIONIER network with over 20000 of digital objects and are the basis for advanced content services provided for educational and scientific users. Examples of such services are distributed metadata search and virtual dynamic collections.

Distributed metadata search is based on periodic metadata synchronization process performed between all digital libraries in the platform. This process is based on OAIPMH protocol implementation in the dLibra framework. Each library collects metadata about all digital objects available in the platform. The metadata is indexed and platform users can search in this metadata for specific digital objects. Currently we are planning to extend search possibilities to include text-based content search. This will be based on OAI-PMH and MPEG-21 DIDL. This platform extension is a subject of current R\&D activities in PSNC's digital library section.

Virtual collections in the PIONIER digital libraries platform are basically collections containing elements from different digital libraries across the platform. There are two kinds of such collections: dynamic and static collections. Virtual dynamic collections are defined by users as conditions that should be met by digital objects metadata. Virtual static collections are based on collection defined by editors in all platform digital libraries. Objects to such collections are added manually by collection editors. When new digital object is published in the platform, it propagates through all digital libraries and all defined virtual collections. This mechanism increases significantly the visibility of digital objects in the PIONIER network.

In our demo we would like to present content services enabled in the PIONIER network, PIONIER digital libraries platform and the software which is used to built the platform. 